\title{
Nutrición, la clave para un mejor rendimiento cognitivo
}

\author{
Nutrition, the key to better cognitive performance \\ Mariana Alba-López ${ }^{a}$, Arantxa Estudillo-León ${ }^{b}$, Jesús G. Jaramillo-Tovar ${ }^{c}$, Olga J. \\ Rodríguez-Antonio ${ }^{d}$, Dafne S. Vázquez-Martínez ${ }^{e}$
}

\begin{abstract}
:
The food we eat has an impact on our body, either negatively or positively. In fact, the importance of a correct nutrition lies in the fact that a considerable amount of metabolic processes of the organism depend on it, as well as the maintenance of good health and it also has an impact on the functioning of the nervous system by giving an increasing cognitive performance. Therefore, it is important to know the nutritional information of what is consumed, such as carbohydrates, vitamins and hydration, and to adopt healthier eating habits.
\end{abstract}

\section{Keywords:}

Foods, brain, cognitive function, nutrient, academic performance.

\section{Resumen:}

Los alimentos que consumimos generan un impacto en nuestro cuerpo, ya sea de manera negativa, como positiva. La importancia de una correcta alimentación radica en que de esta van a depender la considerable cantidad de procesos metabólicos del organismo, el mantenimiento de una buena salud y también tiene un impacto en el funcionamiento del sistema nervioso, dando una creciente en el rendimiento cognitivo. Por ello, es importante conocer la información nutricional de lo que se consume, como lo son los carbohidratos, las vitaminas y la hidratación, y adoptar hábitos alimenticios más saludables.

\section{Palabras Clave:}

Alimentos, cerebro, función cognitiva, nutriente, rendimiento académico.

\section{Introducción}

El propósito de este ensayo es dar a conocer las propiedades de los alimentos y cómo influyen en el proceso de aprendizaje, además de saber cuáles de ellos estimulan al cerebro para obtener un mejor rendimiento académico.

La influencia de los alimentos en la capacidad cerebral se debe a que el cerebro humano es un órgano complejo puesto que siempre se encuentra en estado activo, realizando, creando y desapareciendo conexiones con las neuronas, finalmente de esta forma lleva a cabo funciones vitales para el organismo, por lo cual requiere grandes cantidades de energía para realizar cada uno de estos procesos.

Cuando nos encontramos realizando un proceso cognitivo nuestro cerebro requiere de diversos nutrientes que lo estimulen para efectuar dichas acciones, mejorando su capacidad para procesar, integrar y memorizar cada uno de los conocimientos y aprendizajes adquiridos. Estos nutrientes se van a encontrar en los diferentes grupos de alimentos que consumimos día a día, al saber la diversidad de estos compuestos incluidos en dichos alimentos se pueden integrar en una dieta

\footnotetext{
a Universidad Autónoma del Estado de Hidalgo, https://orcid.org/0000-0002-6853-7619, Email: al454209@uaeh.edu.mx

b Universidad Autónoma del Estado de Hidalgo, https://orcid.org/0000-0001-7674-0441, Email: es351696@uaeh.edu.mx

c Universidad Autónoma del Estado de Hidalgo, https://orcid.org/0000-0001-7324-3351, Email: ja454192@uaeh.edu.mx

d Autor de Correspondencia, Universidad Autónoma del Estado de Hidalgo, https://orcid.org/0000-0003-3874-5852, Email: ro454194@uaeh.edu.mx

e Universidad Autónoma del Estado de Hidalgo, https://orcid.org/0000-0001-5360-3145, Email: va344770@uaeh.edu.mx
} 
equilibrada para así fortalecer el funcionamiento cerebral y neuronal, generando la energía necesaria para lograr estos procesos de manera satisfactoria. Otra ventaja que obtendremos al conocer las propiedades de los alimentos será la disminución de los riesgos de desarrollar enfermedades neurodegenerativas, además de enfermedades en los tejidos nerviosos (vaina de mielina). Dicho esto, los alimentos serán el estímulo que el cerebro requiere, puesto que contienen propiedades como: proteínas, vitaminas, minerales, antioxidantes y grasas, que son convertidas en la fuente de energía que el cerebro necesita. Sin ella, el cerebro no lograría un aprendizaje adecuado y de este modo las personas no tendrían un buen rendimiento académico. De igual manera los nutrientes de dichos alimentos van a influir en microestructuras y macroestructuras cerebrales, así como en la función de los neurotransmisores.

\section{Desarrollo}

Los alimentos a lo largo de la historia han sido la principal fuente de obtención de nutrientes. Podemos definir a la alimentación como "el proceso mediante el cual los seres vivos consumen diferentes tipos de comida" (Diéguez Agraz, 2016) esto con el fin de tener un correcto funcionamiento en el cuerpo humano al ser una de las principales actividades y procesos más esenciales para mantener la homeostasis, en el ser humano se encuentra muy ligada con la supervivencia. Mientras que la nutrición se define como "el conjunto de fenómenos mediante los cuales se obtienen, utilizan y excretan las sustancias nutritivas" (Nutrición Salud Ocupacional, 2019). Dado que los alimentos ingeridos se transforman y se asimilan, es decir, se van a incorporar al organismo gracias a los procesos metabólicos y enzimáticos, para degradarlos y convertirlos en moléculas más pequeñas a los cuales denominamos nutrientes.

Con el transcurso del tiempo la alimentación se ha visto afectada debido a la evolución misma que va de la mano con el cambio en los hábitos, los cuales fueron adquiridos por los pueblos primitivos. Por ende, está modificación en su forma de vida se origina por el nomadismo y sedentarismo, así como los alimentos a los cuales podían acceder en su medio.

Actualmente, al nuevo régimen alimenticio se le han ido incorporando propiedades no nutritivas gracias al capitalismo y al consumismo. En consecuencia esta alimentación ha sido base en los hábitos que demanda la vida actual, propiciada por la facilidad que se tiene de encontrar este tipo de alimentos así como la desinformación nutrimental, desconociendo el impacto negativo que brindamos a nuestro organismo, por ello es importante conocer y destacar cada una de las propiedades que contienen los alimentos, que con el paso del tiempo y extensos estudios, se ha demostrado lo importante que son para la salud y con ello mejorar la cognición o estimulación del cerebro para poder tener un mejor rendimiento académico

Una alimentación adecuada, que aporte al organismo los nutrientes que necesita, combinada con actividad física regular, es clave para gozar de una buena salud. Por el contrario, una alimentación deficiente puede tener efectos muy negativos, reduciendo la capacidad del sistema inmune y restando energía física y mental para desarrollar las actividades básicas con normalidad (Moreno, 2020).

Para comenzar, se tiene que hacer mención sobre cuál es el proceso fisiológico que va a resultar en la estimulación de la "sensación de hambre" (Figura 1).

\section{FUNCIONA.}

\section{LA SENSACIÓN DE HAMBRE}

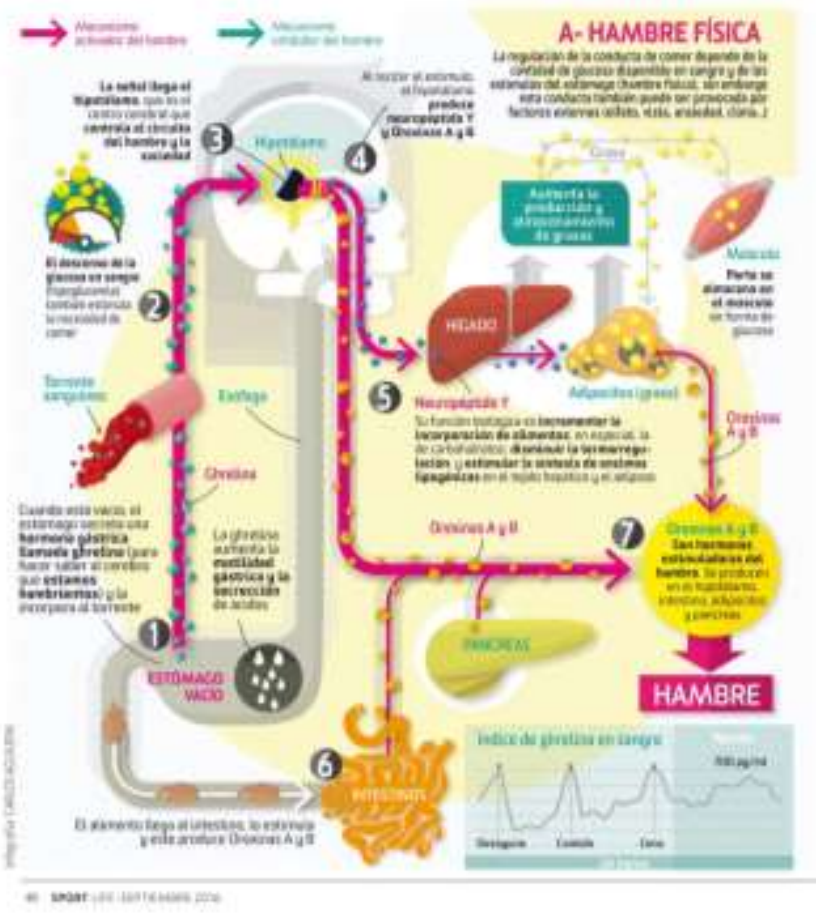

Figura 1. Mecanismo de la sensación de hambre (Aguilera, 2021).

El hambre es regulada por las cantidades de glucosa en la sangre (hipoglucemia) y el hambre física (estímulos 
estomacales), aunque también es producida por los sentidos como la vista, olfato y gusto, además de otros factores como lo es la ansiedad, clima, etc.

Al estar vacío el estómago, secreta la hormona ghrelina, la cual aumenta la motilidad gástrica y la secreción de ácidos, generando que se manden señales al cerebro de que se tiene hambre. Principalmente se lleva a cabo mediante el sistema nervioso central (SNC) por medio del hipotálamo, el cual regula la sensación de hambre y saciedad. Cuando hay afecciones de los núcleos hipotalámicos ventromediales y el paraventricular, hay un desencadenamiento de patologías como lo es la obesidad y la hiperfagia (González Hita et al., 2006).

El estímulo en el hipotálamo provoca la síntesis de neuropéptido $Y$ y orexinas $A$ y $B$. El neuropéptido $Y$ acelera la incorporación de alimentos (carbohidratos), disminuye la termorregulación y estimula la síntesis de enzimas (lipogénicas, encontradas en el tejido hepático y adiposo). Por otra parte, las orexinas $A$ y $B$ estimulan el hambre (producidas en hipotálamo, intestino, adipocitos y páncreas) (González Hita et al., 2006).

La saciedad se produce con la liberación de la leptina en el tejido adiposo, posteriormente pasa al torrente sanguíneo, llegando al hipotálamo y actuando como un inhibidor, dando la señal de que el organismo ya ingirió la cantidad suficiente de comida (Figura 2). Cuando el alimento pasa por el intestino delgado, este produce péptido PYY, para aportar en la inhibición del apetito.

La insulina ayuda a formar tejido adiposo y producir más leptina, inhibiendo la liberación del neuropéptido $Y$, creando una retroalimentación negativa al estímulo del apetito.

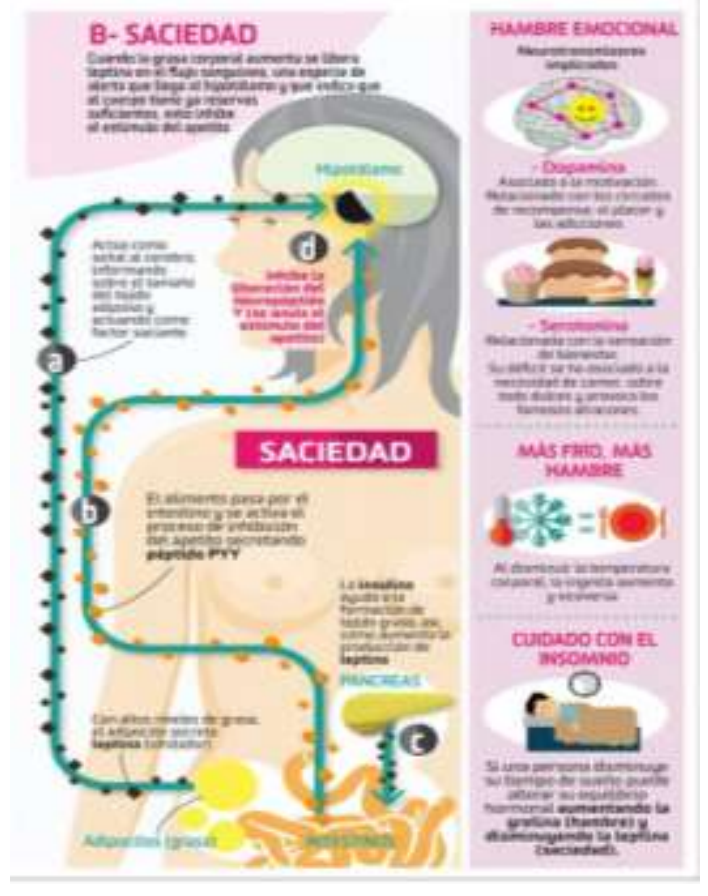

Figura 2. Proceso de la saciedad. (Aguilera, 2021)
En el proceso de la digestión, el alimento es ingresado al organismo después de haber sido ingerido, donde va a ser degradado física y químicamente para que sus componentes puedan ser absorbidos (Carbajal Azcona, 2013).

En este proceso van a intervenir las glándulas salivares, el hígado y el páncreas, los cuales están regulados por mecanismos nerviosos y hormonales.

Por lo tanto, la digestión se basa en dos procesos, uno mecánico y otro químico (Figura 3).

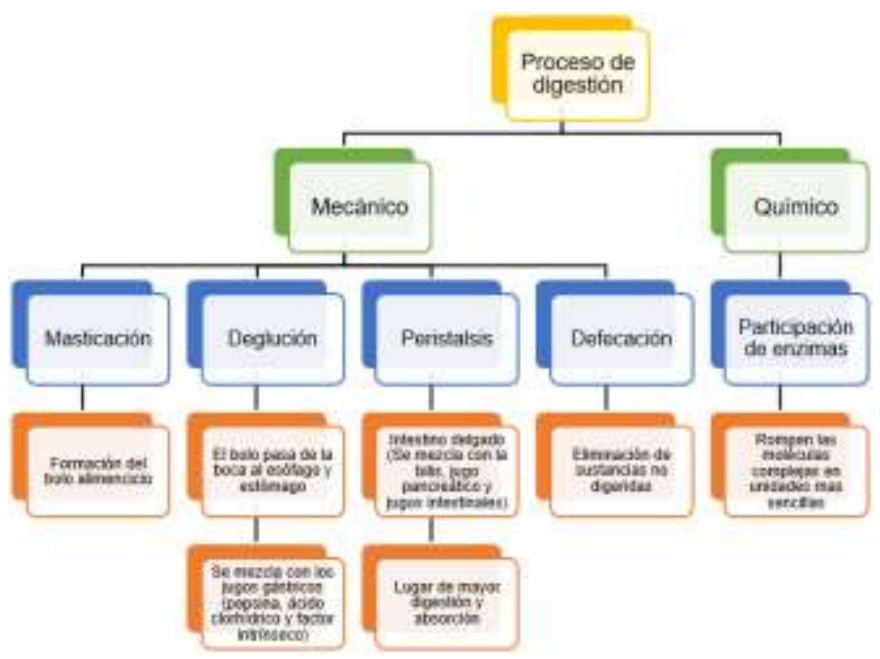

Figura 3. La digestión y sus procesos (mecánico y químico). Elaboración propia basada en el Manual de Nutrición y Dietética por Ángeles Carbajal Azcona de la Facultad de Farmacia por la Universidad Campultense de Madrid. (Carbajal Azcona, 2013)

\section{Proceso de absorción de nutrientes}

Se realiza fundamentalmente en las paredes del intestino delgado, siendo el lugar donde se va a absorber la mayor porción del agua, también se absorben alcoholes, azúcares, minerales y vitaminas hidrosolubles, productos de digestión de proteínas, grasas e hidratos de carbono (Carbajal Azcona, 2013) "De una misma forma las vitaminas liposolubles son absorbidas a la vez con los ácidos grasos" (Páez, 2012).

En el intestino grueso, se reabsorbe una grandiosa porción de agua del remanente que viene del intestino delgado, se acumulan las heces hasta ser expulsadas. Las heces igualmente engloban componentes no absorbidos, residuos celulares, resultado de la constante regeneración de la pared celular. Justo después de ser absorbidos los nutrientes son dirigidos por la sangre a su destino que son las células en las cuales van a ser utilizados los cada uno de estos nutrientes (Tabla 1) (UNED, s. f.). 
Tabla 1. Algunas enzimas del sistema digestivo humano y sus funciones (Cárdenas, 2020).

\begin{tabular}{|c|c|c|}
\hline Organo & Enrimu & Puncikin \\
\hline $\begin{array}{l}\text { GLhndulas } \\
\text { Salivales }\end{array}$ & Tialina & 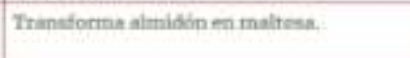 \\
\hline \multirow{2}{*}{ Estómayso } & Seppsina & Trahsifirma pruteinass un aminnobiatos. \\
\hline & $\begin{array}{l}\text { Upaka } \\
\text { pastrira }\end{array}$ & 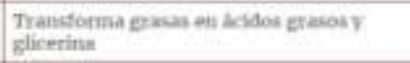 \\
\hline \multirow{3}{*}{ Dimerreas. } & Tripsian & Trensfotuna ptoteinas en eaninoadcisos. \\
\hline & $\begin{array}{l}\text { Anntiasa } \\
\text { pancreitica }\end{array}$ & 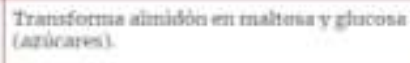 \\
\hline & $\begin{array}{l}\text { Lipose } \\
\text { pancrabitica }\end{array}$ & $\begin{array}{l}\text { Transtorma grases en diddos grntos y } \\
\text { glicerina. }\end{array}$ \\
\hline \multirow{4}{*}{$\begin{array}{l}\text { tnitestina } \\
\text { delexalo }\end{array}$} & Etejnina & Tranitorna pouteinas en aminobicidos. \\
\hline & Maltinsu & Trmintotra matiosa en plucosa \\
\hline & Socatrise & Trantfotma sacinusa en gloconay fructose \\
\hline & Lartasa & Trandforma lactoba en slacosa v sulactosa \\
\hline
\end{tabular}

\section{Función de los nutrientes en la cognición}

"La cognición o desarrollo cognitivo abarca el razonamiento, la memoria, la resolución de problemas y las habilidades de razonamiento" (Head Start ECLKC, 2020).

El término cognición se entiende como el conocimiento alcanzado mediante el ejercicio de las facultades mentales, lo cual implica la facultad o capacidad mental (Rodríguez, J.A, 2007). La facultad intelectual y su base estructurada actúan dentro de los marcos de la percepción, pensamiento, memoria, imaginación y voluntad.

Principales nutrientes que mejoran la capacidad cerebral: Los nutrientes son aportados por la dieta, dependiendo de su consumo dependerá su aprovechamiento metabólico. Las proteínas tienen la capacidad de modular la síntesis de neurotransmisores como la serotonina, dopamina y adrenalina, ya que estos se sintetizan a partir del triptófano y tirosina. Por lo que "la concentración del neurotransmisor serotonina en el cerebro es proporcional a la concentración de triptófano en el plasma y el cerebro" (Vilaplana I Batalla, 2016).

Tabla 2. Alimentos ricos en triptófano y su contenido por mg.. Elaboración propia basada en Los alimentos con más triptófano por Triptomax (Triptomax, 2018) y 10 alimentos ricos en triptófano por Eva Carnero para Saber Vivir TV (Carnero, s. f.)

\begin{tabular}{|l|l|}
\hline Alimentos ricos en triptófano & mg de triptófano \\
\hline Huevo & 156 (clara) \\
& 282 (yema) \\
\hline Pescados & 320 \\
\hline Legumbres (soja) & 532 \\
\hline Frutos secos (nueces) & 265 \\
\hline Frutas (plátano, piña, aguacate) & 17 (plátano) \\
& 19 (aguacate) \\
\hline Lácteos (leche y queso) & 217 \\
\hline Carne magra & 205 \\
\hline
\end{tabular}

Los lípidos y ácidos grasos son la fuente de energía de reserva para el organismo (Tabla 3). Además, son el principal componente de los fosfolípidos de la membrana celular, sus funciones son estimular el desarrollo, la migración y la diferenciación de las células nerviosas.

"Constituyen un sector de la sustancia gris, y de la sustancia blanca, así mismo de los núcleos nerviosos y la sinaptogénesis." (González H., et al., 2015) La suplementación de DHA (ácido docosahexaenoico) durante el embarazo, la lactancia y en los primeros años de vida indica la mejoría del rendimiento mental y el desarrollo psicomotor de los niños, así como del ácido araquidónico, otros ácidos grasos poliinsaturados, ácidos grasos saturados y lípidos complejos. (Martínez García et al., 2018)

La ingesta de dietas con una proporción 5:1 de ácidos grasos omega-6:3, se asocian con una mejor memoria y con un menor riesgo de deterioro cognitivo. (González H., et al., 2015)

\section{La hidratación}

"El agua es un nutriente esencial para el correcto funcionamiento cerebral" (Martínez García et al., 2018) .Una disminución en su ingesta está relacionada con estados de confusión, irritabilidad, letargia además de pérdida de función cognitiva.

Tabla 3. Alimentos que contienen lípidos y ácidos grasos (saturados e insaturados) por $100 \mathrm{~g}$ (PEPSAM-UNNOBA, 2020).

\begin{tabular}{|c|c|c|c|}
\hline Alimento & $\begin{array}{l}\text { Lipldos } \\
\text { (por 100g) }\end{array}$ & $\begin{array}{c}\text { Acldos grasos } \\
\text { eaturados' } \\
\text { (por 100g) }\end{array}$ & $\begin{array}{l}\text { Acidos grasos } \\
\text { insaturados: } \\
(1009)\end{array}$ \\
\hline Aceites y arasas & $99-100 g$ & (variable) & (variable) \\
\hline Mantequilla & $82 \mathrm{~g}$ & 55.1 & $24.9 \mathrm{~g}$ \\
\hline Tocino & $71 \mathrm{~g}$ & $29.4 \mathrm{~g}$ & $40.7 \mathrm{~g}$ \\
\hline Nuez & $63.3 \mathrm{~g}$ & $7.4 \mathrm{~g}$ & $55.8 \mathrm{~g}$ \\
\hline Sobrasada & $61.4 \mathrm{~g}$ & $20.7 \mathrm{~g}$ & $37.8 \mathrm{~g}$ \\
\hline Queso manchego & $30,4 \mathrm{~g}$ & $18.9 \mathrm{~g}$ & $9.8 \mathrm{~g}$ \\
\hline Chocolate negro & $29.2 \mathrm{~g}$ & $16.9 \mathrm{~g}$ & $10.5 \mathrm{~g}$ \\
\hline Costillas de cerdo & $23.6 \mathrm{~g}$ & 9.3 & $12.9 \mathrm{~g}$ \\
\hline Galletas tipo maria & $19 \mathrm{~g}$ & $9.7 \mathrm{~g}$ (variable) & $8.2 \mathrm{~g}$ (variable) \\
\hline Huevos & $11.1 \mathrm{~g}$ & $3.1 \mathrm{~g}$ & $5.7 \mathrm{~g}$ \\
\hline Leche entera & $3.8 \mathrm{~g}$ & $2.3 \mathrm{~g}$ & $1.2 \mathrm{~g}$ \\
\hline Pan blanco & $1.6 \mathrm{~g}$ & $0.4 \mathrm{~g}$ & $0.6 \mathrm{~g}$ \\
\hline Lentejas & $1.2 \mathrm{~g}$ & $0.2 \mathrm{~g}$ & $0.8 \mathrm{~g}$ \\
\hline Pechuga de pollo & $1.2 \mathrm{~g}$ & $0.3 \mathrm{~g}$ & $0.6 \mathrm{~g}$ \\
\hline Arroz & $0.9 \mathrm{~g}$ & $0.2 \mathrm{~g}$ & $0.5 \mathrm{~g}$ \\
\hline Verduras (general) & $<1 \mathrm{~g}$ & (variable) & (variable) \\
\hline
\end{tabular}


Mogollón F. (2021) menciona que "la deshidratación en el encéfalo perjudica la transmisión nerviosa disminuyendo la circulación sanguínea cerebral, lo que puede afectar al rendimiento mental". Se ha observado que personas que contribuyen a tener una correcta hidratación tienen puntuaciones más elevadas en evaluaciones de inteligencia (Mogollón F, 2021).

Una deshidratación leve (2\%) afecta al rendimiento cognitivo (atención, memoria) y al psicomotor (Tovar, 2013).

\subsection{Los minerales}

Encontramos que el hierro es un mineral necesario en el desarrollo de mielinización de las neuronas y en la síntesis de neurotransmisores (catecolaminas y GABA). Su limitada cantidad es habitual en países tanto desarrollados como en crecimiento (Martínez García et al., 2018).

La anemia por deficiencia de hierro se ha relacionado con disminución de la concentración, del razonamiento, de la velocidad de aciertos, del rendimiento, de la memoria, del cálculo, mientras que la administración de suplementos de hierro mejora la capacidad cognitiva (Millán González et al., 2015).

La principal fuente de energía que requiere el cerebro es la glucosa, la cual proviene de los carbohidratos generalmente, como lo son los cereales integrales, frutas, verduras y legumbres (frijol, lenteja, haba, garbanzo, alubias).

Para este desarrollo y un funcionamiento adecuado, el cerebro necesita combustible en forma de glucosa; aproximadamente $5,6 \mathrm{mg}$ de glucosa por cada $100 \mathrm{~g}$ de tejido cerebral por minuto. La glucosa es el único azúcar que alimenta al cerebro, es su principal fuente de energía. En los seres humanos el cerebro requiere aproximadamente el $20 \%$ de la energía derivada de la glucosa, por lo tanto, es necesaria para una función cerebral apropiada (Confederación Española de Alzheimer, 2013).

Para que los hidratos de carbono realicen su compleja función en el cerebro, se necesitan nutrientes esenciales los cuales son los ácidos grasos, vitaminas y minerales. Si llegara a existir una carencia de al menos uno de estos elementos, podría existir una afección en el sistema nervioso. El cerebro junto al sistema nervioso tienen una estrecha dependencia con las grasas, pero no cualquier tipo de grasas, son grasas llamadas esenciales, como es el caso del omega 6 y omega 3 (Piñeiro Lamas \& Díaz Lorenzo, 2016, pág. 26).

Los principales nutrientes necesarios para el sistema nervioso son los siguientes (Tabla 4): Tabla 4 Nutrientes esenciales para el cerebro y su acción (Vilaplana I
Batalla, 2016).

\begin{tabular}{|c|c|}
\hline Nutriente & Acrión \\
\hline Fósforo & $\begin{array}{l}\text { Contribuye al funcionamiento normul de las } \\
\text { membranas celulues. }\end{array}$ \\
\hline Ácido docosaheranenoico & $\begin{array}{l}\text { Contribuye a mantener ef funcionamiento normal } \\
\text { del cermbre. }\end{array}$ \\
\hline Yodo & $\begin{array}{l}\text { Contribuye al funcionamiento normal del sistema } \\
\text { nervioco y a la hunción coinnitiva nomal }\end{array}$ \\
\hline Axido pantotenico & Contribuye al rendimiento intelectual normal \\
\hline Ácide folice. & Contribuye a ina funcios pskolbgica normal. \\
\hline Calcio & $\begin{array}{l}\text { Cocitribuye ai funcionamiento narmal de is } \\
\text { neurntransmision. }\end{array}$ \\
\hline $\begin{array}{l}\text { Biotine, flamina, vitarnina f65. } \\
\text { vitamina } C\end{array}$ & $\begin{array}{l}\text { Contribioyen al funcianamiento nomal del sistema } \\
\text { nervioso y a una funclan pricologica normal. }\end{array}$ \\
\hline $\begin{array}{l}\text { Cobore, magnesio, potacio, } \\
\text { vtamana } 12 \text {, vitaruina } \$ 12 \text {, niacina }\end{array}$ & $\begin{array}{l}\text { Contribuyen al funcionsmiento nomal del sintema } \\
\text { nerviaso. }\end{array}$ \\
\hline Agua & $\begin{array}{l}\text { Contribuye a mantener las funcones fisicas y } \\
\text { cognitivas nomales. }\end{array}$ \\
\hline Hierro, zine & Contribuyen a la función cogritiva normal. \\
\hline
\end{tabular}

\section{Vitaminas B}

Estas vitaminas son de importancia debido a su función como disminuidor de los niveles de ósmosis presentes en la sangre, por tanto, son un "aminoácido considerado como factor en el riesgo vascular" (Richly et al., 2014).

"Las vitaminas del grupo B desempeñan una función clave en asegurar que el cerebro alcance todo su potencial" (Nutrir la mente: vitaminas B para un cerebro bien alimentado, 2021).

Grupo de nutrientes que influyen en el buen funcionamiento del cerebro y en el sistema nervioso:

- Vitamina B1 o tiamina, los alimentos que la aportan son: Soja fresca, germen de trigo, carnes, pescados, frutos secos, legumbres y en especial la avena.

"Es fundamental para el proceso de
transformación de azúcares y cumple una
importante labor en la conducción de los impulsos nerviosos y en el metabolismo del oxígeno. Pérdida de memoria, deficiencias cognitivas, 
confusión mental, dificultades con el habla, irritabilidad, apatía, temblores" (Ratner , 2017).

- Vitamina B6 o piridoxina, los alimentos que la aportan son: "ternera, hígado, cerdo, aves, cordero, mariscos, hígado de pescado, yema de huevo, lácteos, cereales integrales y sus derivados, leguminosas, germen de trigo, levadura de cerveza" (Ruiz de las Heras , 2021).

Interviene en diversos aspectos del metabolismo y la biosíntesis de diversos neurotransmisores entre ellos, la serotonina a partir de triptófano-, y en la formación de las vainas de mielina de las neuronas, aislamiento necesario para que las neuronas y las fibras nerviosas puedan transmitir correctamente las señales $\mathrm{y}$, por tanto, las órdenes de nuestro cerebro a los músculos del cuerpo. Su aporte deficiente puede causar irritabilidad, nerviosismo, fatiga e incluso depresión (Vilaplana I Batalla, 2016).

- Vitamina B12 o cianocobalamina, los alimentos que los proporcionan provienen de origen animal, por ejemplo: sardinas, atún, solomillo, pate de hígado, huevos y queso.

"Es una vitamina hidrosoluble esencial que ayuda a mantener sanas las neuronas para el correcto funcionamiento del cerebro y del sistema nervioso, así como para la formación de los glóbulos rojos de la sangre y de diversas proteínas fundamentales para el organismo" (Hospital Universitari General de Catalunya, 2020).

\section{Aminoácidos}

La principal función de los aminoácidos es la de sintetizar a los neurotransmisores, principalmente las catecolaminas y serotonina, debido a que nos van a brindar un suministro de esta y no se encuentra en grandes porciones en nuestro cerebro.

La producción de los mediadores (catecolaminas y serotonina) va a depender demasiado de la cantidad de proteínas que vamos a ingerir en una dieta diaria, la cual va a cubrir en el rendimiento celular o cognitivo cerebral era cerebral no celular (Castillo Sarmiento \& RodríguezMartín, 2001).

El cerebro contiene líquido intersticial el cual está constituido en su mayor parte de péptidos (Ibáñez Benages, 2009) ,debido a que va a suministrar las proteínas a nuestro sistema nervioso como lo puede ser la taurina glutamina carnitina y otros productos de origen amino.

\section{Melatonina}

"Esta es conocida a nivel mundial como la hormona del sueño, pues su función básica es regular el ciclo de sueño y del ritmo circadiano, logrando un descanso completo y recuperador" (Carpintero Angulo, 2021). Esta desempeña diversas funciones biológicas muy específicas como, por ejemplo:

- Re sincroniza el ritmo circadiano sueño vigilia, favorece la reparación del sueño.

- Actúa sobre las funciones de la retina.

- Controla el crecimiento tumoral.

- Participa en la liberación de radicales libres y regulación de la tensión arterial.

La síntesis y liberación de melatonina se estimula con la oscuridad y se suprime con la luz, su precursor es el aminoácido triptófano, que se convierte en serotonina gracias a la actividad mediada por las enzimas serotonina-acetiltransferasa e hidroxiindol-Ometiltransferasa, que convierten serotonina en melatonina (Reyes Prieto et al., 2009).

Se debe tener en cuenta que "La concentración de serotonina en el cerebro es proporcional a la concentración de triptófano en el plasma y el cerebro" (Contreras Roura et al., 2015) a su vez, la serotonina se convierte en la molécula $\mathrm{N}$-acetil serotonina, precursora de la melatonina.

\section{Triptófano}

Uno de los principales aminoácidos que requiere nuestro cerebro es el triptófano debido a que es un modulador del apetito y la saciedad (Ibáñez Benages, 2009, pág. 5). Otra de sus funciones es que funge como intermediario entre el sueño, la sensibilidad al dolor y la regulación 
sanguínea, de igual manera en las alteraciones en nuestro comportamiento.

Por lo tanto, es uno de los aminoácidos más esenciales debido a que el cuerpo no puede producirlo por sí mismo, por lo cual debemos incluirlo en nuestra dieta diaria para que el organismo fabrique las proteínas que requiere. Además, es necesario para la síntesis de melatonina y serotonina (Ramos, 2021).

La importancia del triptófano radica en que es un aminoácido (unidad estructural de las proteínas) presente en alimentos de origen animal y su consumo mejora la atención, la memoria visual y el aprendizaje (González García et al., 2011).

La niacina (vitamina B3), puede ser sintetizada a partir del aminoácido triptófano, donde $60 \mathrm{mg}$ de triptófano puede producir $1 \mathrm{mg}$ de niacina (Allen et al., s. f.)

"La OMS declara que un adulto requiere $3,5 \mathrm{mg}$ de triptófano por kg de peso al día. Para calcular la cantidad a ingerir, se acepta que, de promedio, las proteínas de la dieta contienen un mínimo del $1 \%$ de triptófano" (Vilaplana I Batalla, 2016, pág. 20).

De forma frecuente las deficiencias que se presentan de niacina (vitamina B3) y triptófano se combinan con la de vitamina B6, causando daño periférico en el cerebro (Frye \& Jabbour, 2021).

\section{Serotonina y función cerebral}

Al consumir alimentos ricos en triptófano en nuestra dieta, este aminoácido cumple con la función de ser el principal precursor de la serotonina (Segura, 2020).

La serotonina es un neurotransmisor que tiene unas funciones muy importantes a nivel neuronal (Trueta \& Cercós, 2012).

- Modera la temperatura corporal.

- Regula la actividad motora junto con las funciones perceptivas y cognitivas.

- Controla los parámetros de densidad ósea.

- Interviene en la regeneración hepática.

- Regula el sueño, los estados de ánimo, las emociones y los estados depresivos.

- Interviene en el funcionamiento vascular y la frecuencia cardiaca.

- Regula la secreción de hormonas.

- Previene desequilibrios mentales como la esquizofrenia, trastorno obsesivo compulsivo o autismo infantil.

- Favorece la proliferación linfocitaria.

- Regula el humor y comportamiento.
El triptófano favorece la síntesis de serotonina, la serotonina es un neurotransmisor cuya distribución es proporcional tanto en el plasma como en el cerebro, ya que las plaquetas almacenan y capturan la serotonina, causando un acumulo y transporte de este neurotransmisor al ser estimulado por el triptófano (Moreno et al., 2006).

El metabolismo del triptófano es complejo y tiene muchos procesos. Requiere de una cantidad adecuada de vitamina B6 y magnesio para desempeñar su función de manera adecuada. La función de la vitamina B6 está ligada a la conversión de triptófano en serotonina (Vilaplana I Batalla, 2016, pág. 20).

Martínez García et al. (2018) encontró que los niveles bajos de serotonina se asocian con la disminución del aprendizaje, el razonamiento y la memoria

\section{Antioxidantes}

Los antioxidantes son sustancias naturales que las vamos a encontrar en los alimentos de origen vegetal. Estos van a ser demasiado positivos para nuestra salud puesto que como su nombre lo indica, son antioxidantes que pueden repeler el daño de envejecimiento de nuestras células del cuerpo (lbáñez Benages, 2009).

Debido a que es una de las principales causas del deterioro cognitivo es ocasionado por un daño irreversible del cerebro al ser propenso a dañarse por los radicales libres que van a contener la unión de las moléculas. Por eso, los antioxidantes, al secuestrar radicales libres y eliminarlos de la circulación pueden tener un efecto preventivo frente a la peroxidación lipídica (Ibáñez Benages, 2009).

El deterioro cognitivo se ha asociado con estrés oxidativo. Muchos autores indican que podría prevenirse con el consumo de antioxidantes (vitaminas $C$ y $E$, betacarotenos, cinc y selenio). La vitamina $C$ interviene en la protección del tejido nervioso del estrés oxidativo. Mientras que, la deficiencia de cinc está relacionada con el TDAH en niños, problemas de memoria y aprendizaje en adolescentes y estrés, depresión y deterioro cognitivo en adultos y ancianos (Martínez García et al., 2018).

Los antioxidantes también los produce en el cuerpo, pero de igual manera no pueden ser suficientes para tener el sistema de defensa tan alto por ellos igual manera muy importante ingerirlos (Martínez García et al., 2018). 


\section{Polifenoles}

Éstos son antioxidantes presentes en alimentos de origen vegetal como lo son los frutos rojos, el resveratrol, las catequinas, las procianidinas y los licopenos. Su principal función es reducir el daño neuronal producido por el estrés oxidativo

Todos estos antioxidantes deben ingerirse en dosis adecuadas debido a que podría causar una elevada presencia de metales como consecuencia inducen a una pérdida de sus propiedades anti oxidativas ganando propiedades prooxidantes (González García et al., 2011).

\section{Los ácidos grasos}

Los ácidos grasos se dividen en dos grupos según sus características estructurales: los ácidos grasos insaturados. Dependiendo de cuantos enlaces doble tengan y su posición forman el omega-9, omega-6 y omega-3. (Pascual Chagman, s. f.)

Nuestro organismo no puede introducir insaturaciones en las posiciones que forman el omega- 6 y el omega-3, por lo tanto, existen dos precursores, los ácidos linoleico "(C18:2n6) y alfa linolénico (C18:3n3), a los que llamamos esenciales y que deben ser incorporados por la dieta. Esta última debe contenerlos en proporciones bien determinadas, ya que su carencia o desbalance en la ingesta producen serias alteraciones metabólicas" (González et al., 2015).

\section{Omega-3}

Es un conjunto de ácidos grasos poliinsaturados, estos son esenciales debido a que el organismo no los puede producir por sí mismo por ende deben ser ingeridos en la dieta diaria para poder completar el desarrollo cognitivo. Su importancia radica debido a que van a formar parte de las membranas de las células del cerebro que son las neuronas, por este motivo resulta clave el mantenerlos en nuestra dieta diaria, principalmente se encuentra en pescados como lo es el salmón rosado, la trucha, atún, y por último mariscos, también en las frutas secas como lo son las nueces, almendras y los aceites vegetales por ejemplo el de soja o aceite de canola cuya importancia radica en que, una vez que van a ingresar al organismo el ácido linolénico que lo contienen va a convertirse en un catalizador de las enzimas del ácido docosahexaenoico (Richly et al., 2014).

\section{Hidratos de carbono.}

Los hidratos de carbono tienen una función importante debido a que es un combustible por excelencia de todas las células especialmente de las células nerviosas debido a que el único hidrato de carbono que se va a utilizar en el cerebro es la glucosa, por tanto, se produce más energía en presencia del oxígeno y esto da como resultado un aumento o incremento del rendimiento energético que va a precisar como un oxidante (Castillo Sarmiento \& Rodríguez-Martín, 2001).

La ingesta mínima diaria se cifra en 1,4 g por kilogramo al día de glucosa para el buen desarrollo de las funciones cerebrales (Ibáñez Benages, 2009). Al verse limitadas las reservas de glucosa converge una afección muy importante por la sensibilidad de la falta de glucosa es la hipoglucemia.

Mientras que al encontrar elevadas concentraciones de glucosa en la sangre van a contribuir a preservar la función cognitiva durante el estrés por eso es importante el ayuno intermitente en algunas ocasiones.

\section{Conclusiones}

Se debe hacer conciencia sobre los alimentos que se ingieren y conocer la utilidad nutricional que aportan en nuestro organismo. La principal importancia de una buena alimentación se debe a la función cognitiva, debido a que ayuda a prevenir enfermedades neurodegenerativas como el Alzheimer.

Una de las principales consecuencias que se han encontrado en diversos estudios es la falta del aporte de sustratos energéticos al cerebro que comprometen la supervivencia neuronal y desencadenan procesos de neurotoxicidad debido al incremento de la neurotransmisión del glutamato. Al ser este de los principales neurotransmisores, su disminución afecta la función cognitiva, además de una producción deficiente de ATP en las células del sistema nervioso central, impidiendo la recaptación de glutamato en la terminal sináptica de los astrocitos, así como el proceso dinámico de las células neuronales para el transporte de información hacia su botón sináptico.

Con el aumento de la esperanza de vida se ha incrementado la cantidad de personas con deterioro cognitivo y demencia en las últimas décadas. Por ello la importancia de mantener una alimentación balanceada, de la cual el cerebro requiere todos los nutrientes esenciales que serán necesarios para su correcto y sano o normal funcionamiento.

\section{Referencias}

1. Aguilera, C. (10 de septiembre de 2021). SportLife. Obtenido de SportLife: https://www.sportlife.es/adelgazar/como-funcionasensacion-hambre_198023_102.html

2. Allen, L., de Benoist, B., Dary, O., Hurrell, R., \& Organización Mundial de la Salud. (s. f.). Guías para la fortificación de alimentos con micronutrientes. Organización Mundial de la Salud. 
https://apps.who.int/iris/bitstream/handle/10665/255541/97892435940 19-spa.pdf

3. Carbajal Azcona, Á. (2013). Universidad Complutense de Madrid. Obtenido de Universidad Complutense de Madrid: https://www.ucm.es/data/cont/docs/458-2013-07-24-cap-13-digestionabsorcion.pdf

4. Cárdenas, J. (7 de junio de 2020). Leer Ciencia. Obtenido de https://leerciencia.net/aparato-digestivo-humano-estructura-y-funcion/

5. Carnero, E. (s.f.). Saber Vivir TV. Recuperado el 01 de Octubre de 2021, de Saber Vivir https://www.sabervivirtv.com/nutricion/alimentos-ricostriptofano_1663

6. Carpintero Angulo, A. (31 de julio de 2021). Farmacia Angulo Blog. Recuperado el 14 de octubre de 2021, de Farmacia Angulo Blog: https://nutricionyfarmacia.es/blog/salud/bienestar/melatonina-lahormona-sueno/

7. Castillo Sarmiento, C. A., \& Rodríguez-Martín, B. (Eds.). (2001). Alimentación y cerebro. Universidad de Castilla- La Mancha. Published. https://doi.org/10.18239/atenea_2021.23.00

8. Confederación Española de Alzheimer. (20 de noviembre de 2013). Confederación Española de Alzheimer. Obtenido de https://www.ceafa.es/es/que-comunicamos/noticias/el-cerebronecesita-constantemente-glucosa-para-su-correcto-funcionamiento

9. Contreras Roura, J., Alonso Jiménez, E., \& Fuentes Smith, L. E. (Enero-Abril de 2015). Revista CENIC Ciencias Biológicas. Recuperado el 3 de Octubre de 2021, de La concentración de serotonina en el cerebro es proporcional a la concentración de triptófano en el plasma $\quad y$ el cerebro: https://www.redalyc.org/pdf/1812/181238817001.pdf

10. Diéguez Agraz, C. (2016). Decídete a tener una vida sana. México, D.F. : Selector.

11. Frye, R. E., \& Jabbour, S. A. (2021, 19 abril). Pyridoxine Deficiency. Medscape. Recuperado 14 de octubre de 2021, de https://emedicine.medscape.com/article/124947-overview

12. González Garcia, K. L., Valdés Iglesias, O., Laguna, A., Díaz Martínez, M., \& González Lavaut, J. A. (2011). Efecto antioxidante y contenido polifenólico de Syringodium filiforme (Cymodoceaceae). Revista de Biología Tropica, 59(1), 465-472. https://www.redalyc.org/articulo.oa?id=44918841040

13. González, H., \& Visentin, S. (24 de Noviembre de 2015). Nutrientes y neurodesarrollo: Lípidos. Actualización. Recuperado el 03 de Octubre de 2021, de Sociedad Argentina de Pediatría : https://www.sap.org.ar/docs/publicaciones/primero/2016/Act_Gonzal ez_anticipo_12-9-16.pdf

14. González Hita, M. E., Ambrosio Macias, K. G., \& Sánchez Enríquez, S. (2006, diciembre). Regulación neuroendócrina del hambre, la saciedad y mantenimiento del balance energético. Medigraphic. https://www.medigraphic.com/pdfs/invsal/isg-2006/isg063i.pdf

15. Head Start ECLKC. (27 de julio de 2020). Obtenido de https://eclkc.ohs.acf.hhs.gov/es/preparacion-escolar/effectivepractice-guides/cognicion

16. Hospital Universitari General de Catalunya. (17 de septiembre de 2020). Quirón Salud. Recuperado el 13 de octubre de 2021, de Quirón Salud: https://www.quironsalud.es/es/comunicacion/notasprensa/importancia-vitamina-b12-organismo

17. Ibáñez Benages, E. (02 de Mayo de 2009). Nutrición Hospitalaria Suplementos. Recuperado el 2021, de Nutrición Hospitalaria Suplementos: https://www.redalyc.org/pdf/3092/309226754002.pdf
18. Martínez García , R. M., Jiménez Ortega, A. I., López-Sobaler, A. M., \& Ortega, R. M. (2018). SCIELO. Obtenido de SCIELO: https://scielo.isciii.es/pdf/nh/v35nspe6/1699-5198-nh-35-nspe600016.pdf

19. Millán González, A., Martínez García , R., Serrano Parra, D., \& Nieto López, M. (diciembre de 2015). Nutrición Hospitalaria. Obtenido de Nutrición https://www.redalyc.org/pdf/3092/309243334064.pdf

20. Mogollón, F. (16 de abril de 2021). ÁGORA. Recuperado el 03 de octubre de 2021, de ÁGORA: https://agora.uniandes.edu.co/comoalimentar-nuestro-cerebro/

21. Moreno, J., Campos, M. G., Lara, C., López, G., Pavón, L., Hernández, M. E., Sentíes, H., González-Olvera, J., Torruco, M., Arango, I., Heinze, G., \& Torner, C. (2006). Tryptophan and serotonin in blood and platelets of depressed patients: Effect of an antidepressant treatment. Scielo. Recuperado 13 de octubre de 2021, de http://www.scielo.org.mx/scielo.php?script=sci_arttext\&pid=S018533252006000400001

22. Moreno, M. (2 de enero de 2020). Veritas Intercontinental. Obtenido de Veritas Intercontinental: https://www.veritasint.com/blog/es/losbeneficiosos-efectos-en-la-salud-de-una-dieta-equilibrada/

23. Nutrición Salud Ocupacional. (25 de junio de 2019). Respira Ocupacional. Obtenido de Respir Ocupacional: https://respirasac.com/home/nutricion-salud-ocupacional-2/

24. Nutrir la mente: vitaminas B para un cerebro bien alimentado. (2021, 15 marzo). Cono Sur BAYER. Recuperado 13 de octubre de 2021, de https://www.conosur.bayer.com/es/nutrir-la-mente

25. Páez, D. (24 de febrero de 2012). PÁEZ DOCTORA. Recuperado el 03 de octubre de 2021, de PÁEZ DOCTORA: https://doctorapaez.com/2012/02/alimentacion-y-nutricion/

26. Pascual Chagman, G. J. (s. f.). Innovaciones tecnológicas en la producción de aceites con alto contenido en omega 3. Repositorio CONCYTEC.

http://repositorio.concytec.gob.pe/bitstream/20.500.12390/365/15/2_I nnovaciones_en_producci\%C3\%B3n_de_aceites_alto_omega_3.pdf

27. PEPSAM-UNNOBA. (2020). UNNOBA. Obtenido de UNNOBA: https://www.unnoba.edu.ar/wpcontent/uploads/2020/05/alimentaci\%C3\%B3n-clase-8-archivo-2.pdf

28. Piñeiro Lamas, R., \& Díaz Lorenzo, T. (2016). Neuronutrición y trastornos cognitivos en niños y niñas. Ciudad de México. Obtenido de http://partidodeltrabajo.org.mx/2017/wpcontent/uploads/2017/07/Libro-Neuronutricion-final-imprenta.pdf

29. Ramos, M. (11 de mayo de 2021). Microsismos. Recuperado el 14 de octubre de 2021, de Microsismos: https://www.publico.es/psicologia$\mathrm{y}$-mente/beneficios-y-peligros-del-triptofano/

30. Ratner, R. (2017). Déficit de vitamina B. Nutrición y Salud , 70-71.

31. Reyes Prieto, B. M., Velázquez Paniagua, M., \& Prieto Gómez, B. (2009). Melatonina y neuropatologías. Revista de la Facultad de Medicina UNAM, 52(3), 105-109. https://www.medigraphic.com/pdfs/facmed/un-2009/un093d.pdf

32. Richly, P., Vilaro, S., O`Neill, S., Bustin, J., \& Martínez, D. (2014). Comida para un cerebro saludable ( $1 .^{\text {a }}$ ed.). FINECO. https://www.ineco.org.ar/wp-

content/uploads/comida_para_un_cerebro_saludable.pdf

33. Rodríguez, J. A. (15 de noviembre de 2007). Cognición y ciencia cognitiva. Obtenido de Cognición y ciencia cognitiva: http://cmap.upb.edu.co/rid=1204129145046_1169029526_15492/cgn 01.pdf 
34. Ruiz de las Heras, A. (29 de marzo de 2021). Webconsultas. Obtenido de Webconsultas: https://www.webconsultas.com/dieta-ynutricion/dieta-equilibrada/micronutrientes/vitaminas/vitamina-b61817

35. Segura, A. (16 de diciembre de 2020). La Vanguardia. Recuperado el 2021 de octubre de 13, de La Vanguardia: https://www.lavanguardia.com/comer/tendencias/20201216/6118761/ razones-deberias-consumir-alimentos-ricos-triptofano-precursorserotonina.html

36. Tovar, J. (03 de diciembre de 2013). EFE SALUD. Obtenido de EFE SALUD: https://www.efesalud.com/cuidado-con-la-deshidratacionun-2-afecta-al-rendimiento-y-las-habilidades-cognitivas/

37. Triptomax. (14 de febrero de 2018). Triptomax. Recuperado el 1 de Octubre de 2021, de Triptomax: https://www.triptomax.com/blog/alimentos-ricos-triptofanoserotonina/

38. Trueta, C., \& Cercós, M. G. (2012). Regulación de la liberación de serotonina en distintos compartimientos neuronales. Salud Mental, 35, 435-443. https://www.redalyc.org/articulo.oa?id=58225155011

39. UNED Facultad de Ciencias, Nutrición y Dietética. (s.f.). UNED Recuperado el 01 de Octubre de 2021, de UNED: https://www2.uned.es/pea-nutricion-y-dieteticaI/guia/guia_nutricion/el_proceso_de_la_nut.htm

40. Vilaplana I Batalla, M. (2016). Nutrición. Alimentación y proteínas. Farmacia Profesional, 17-20. Obtenido de https://www.elsevier.es/esrevista-farmacia-profesional-3-pdf-X0213932416603690 